\title{
A New Cluster Head Selection Algorithm
}

\author{
LUO Qin \\ College of Information Engineering, Jiangxi College of Technology, Jiangxi Nanchang 330098
}

Keywords: Fuzzy Logic; Residual Energy ;Distribution Density; Threshold

\begin{abstract}
The LEACH protocol selects cluster head nodes randomly in round way, which causes some problems, such as the network energy consumption is not balanced, the network cycle is not ideal. This paper presents a cluster head selection algorithm based on fuzzy logic. Firstly, it has some important input variables such as residual energy, density and distance to the base station. Secondly, it makes some fuzzy rules. Then it calculates a probability of cluster head through the fuzzy inference system. Finally, it improves the threshold value by the probability. Simulation results show that the algorithm can make the network more balanced energy consumption, effectively prolong the network life cycle compared with the LEACH protocol.
\end{abstract}

\section{Introduction}

LEACH (low-energy adaptive clustering hierarchy) [1] proposed by Heinzelman et al, which is the earliest in the cluster based routing protocols for WSN. LEACH using random election of cluster head nodes, which causes the energy consumption of each node imbalance. It is a research focus that selecting the optimal cluster head communicates with the base station through the concept of fuzzy logic introduced in LEACH.

In recent years, there are some cluster head selection algorithms for wireless sensor network based on fuzzy logic at home and abroad. For example, it holds that there are the residual energy of nodes and the number of neighbor nodes as an important factor in the cluster head selection in reference [2]. It introduces the speed of energy consumption as an important factor in the cluster head selection in reference [3]. It introduced node communication flow as an important factor for cluster head selection in reference [4].It also introduced the average distance of cluster head and the neighbor node as cluster head selection in reference [5].

This paper presents the FLCHS algorithm, which is stated that the residual energy of nodes, the selected node distribution density and the node to the base station distance are the important factor for a node to become a cluster head node. It computes the probability of becoming cluster head for each node through the fuzzy inference rules. According to the probability, selecting the optimal cluster head, so the cluster head has enough energy to ensure data transmission. The FLCHS algorithm can prolong the network life cycle.

\section{FLCHS Algorithm}

The $\mathbf{N}$ sensors are randomly deployed in $\mathbf{M} * \mathbf{M}$, and based on the following assumptions:

(1)The base station is fixed and located far from the sensors.

(2) The initial energy of nodes are the same;

(3) Nodes can perceive its residual energy;

(4) Nodes can perceive its geographical position; 
(5) The base station can calculate the density for every node around by its geographical location;

(6) The base station can calculate the distance to each node through its geographical location;

(7) Wireless communication between nodes is symmetrical.

(8)The energy model uses the first order radio model

\section{The fuzzification of input and output variables}

(1) The residual energy: energy (j) represents the residual energy of node j.It is divided into low , medium, high. Among them, low adopts the right trapezoid membership function, medium uses triangle membership function, high uses the left trapezoid membership function (Fig. 1).

(2) The distribution density (j) represents the adjacent nodes of node $\mathrm{j}$ in the circular area with center at node $\mathrm{j}$ and radius equal to $\sqrt{M^{2} /\left(\pi^{*} N^{*} q\right)} \cdot \operatorname{density}(\mathrm{j})=\sum_{i=1}^{m} N(i), \mathrm{N}(\mathrm{i}) \in \operatorname{NeighborSet}(\mathrm{j}) 。$ It is divided into three grades, sparse, medium, dense .Among them, sparse adopts the right trapezoid membership function, medium uses triangle membership function, dense uses the left trapezoid membership function(Fig. 2).

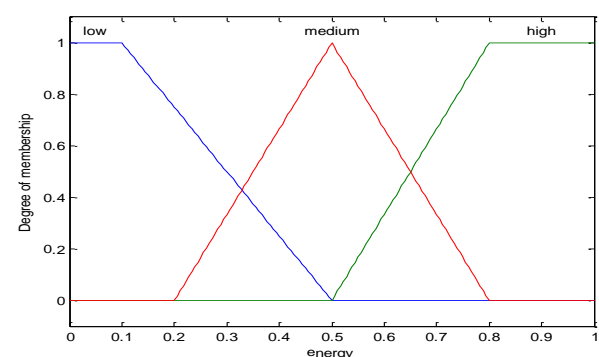

Fig. 1 membership function of residual energy

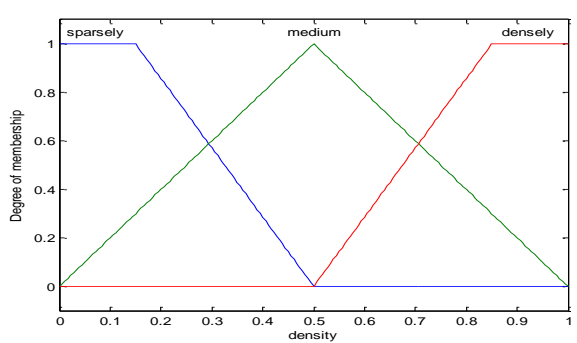

Fig. 2 membership function of distribution density

(3) The distance to the base station: distance (j) represents the distance to the base station. It is divided into near, far, medium. Among them, near uses the right trapezoid membership function, medium uses triangle membership function, far uses the left trapezoid membership function( Fig. 3).

(4) The probability of nodes become cluster head:probability(j) represents the probability. It is divided into very low 、 low 、 little low 、 little middle、 middle、 higher middle、 little high 、 high 、 very high(Fig. 4).

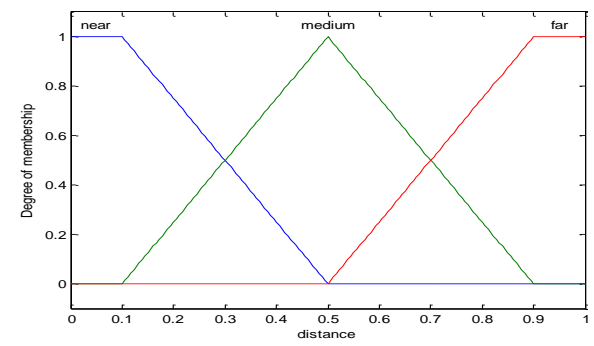

Fig. 3 the distance to base station

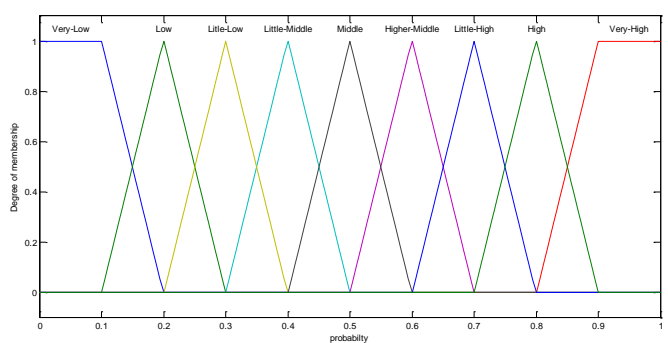

Fig. 4 the probability of selected cluster head 
This paper uses Mamdani model[4], makes 27 fuzzy rules, the specific rules are as follows:

Table 1 fuzzy rules table

\begin{tabular}{|c|c|c|c|c|c|c|c|c|c|}
\hline & energy & density & distance & probability & & energy & density & distance & probability \\
\hline 1 & high & dense & near & very high & 15 & medium & medium & far & $\begin{array}{l}\text { little } \\
\text { middle }\end{array}$ \\
\hline 2 & high & dense & medium & very high & 16 & medium & spare & near & $\begin{array}{l}\text { little } \\
\text { middle }\end{array}$ \\
\hline 3 & high & dense & far & high & 17 & medium & spare & medium & $\begin{array}{c}\text { little } \\
\text { middle }\end{array}$ \\
\hline 4 & high & medium & near & high & 18 & medium & spare & far & $\begin{array}{c}\text { little } \\
\text { middle }\end{array}$ \\
\hline 5 & high & medium & medium & little high & 19 & low & dense & near & $\begin{array}{c}\text { little } \\
\text { middle }\end{array}$ \\
\hline 6 & high & medium & far & little high & 20 & low & dense & medium & $\begin{array}{c}\text { little } \\
\text { middle }\end{array}$ \\
\hline 7 & high & spare & near & little high & 21 & low & dense & far & little low \\
\hline 8 & high & spare & medium & little high & 22 & low & medium & near & little low \\
\hline 9 & high & spare & far & middle & 23 & low & medium & medium & little low \\
\hline 10 & medium & dense & near & $\begin{array}{c}\text { higher } \\
\text { middle }\end{array}$ & 24 & low & medium & far & little low \\
\hline 11 & medium & dense & medium & $\begin{array}{c}\text { higher } \\
\text { middle }\end{array}$ & 25 & low & spare & near & low \\
\hline 12 & medium & dense & far & middle & 26 & low & spare & medium & very low \\
\hline 13 & medium & medium & near & middle & 27 & low & spare & far & very low \\
\hline 14 & medium & medium & medium & middle & & & & & \\
\hline
\end{tabular}

Fuzzy logic inference process contains three input variables, 27 fuzzy rules and an output variable. It uses the centroid method[4] to defuzzify, output the probability value of becoming a cluster head. For example, if energy $=0.3$, density $=0.5$, distance $=0.1$, then probability $=0.385$.

Improved threshold value by the probability of elected as cluster head,such as the formula (1):

$$
\mathrm{T}(\mathrm{n})= \begin{cases}\frac{q}{1-q \times\left\{r \bmod \left(\frac{1}{q}\right)\right\}} & \\ 0 & \text { other }\end{cases}
$$

Among them,rs represents the rounds that the node hasn't be selected cluster head continuous rounds before, If selected as the cluster head, then the rs is set to 0 . When $r s=1 / p, T$ (n) will increase.

Algorithm description. Step1: node $\mathrm{j}$ according to itself residual energy, density distribution and the distance to the base station, uses fuzzy logic reasoning, to calculate the probability of node $\mathrm{j}$ selected as the cluster head value, expressed as probability (j);

Step2: according to the formula 1, calculate the threshold $T(j)$ of node $j$;

Step3: node $\mathrm{j}$ generates a random number between 0 and 1 , expressed as rand $(\mathrm{j})$; Step4: if rand $(\mathrm{j})<\mathrm{T}(\mathrm{j})$, node $\mathrm{j}$ will be selected as the cluster head node, and broadcast message to other nodes, executing step5 else jumping to step6;

Step5: node $\mathrm{j}$ receives a join request from ordinary nodes, it assigns TDMA slot to the members of the cluster.

Step6: node $\mathrm{j}$ is waiting until receiving a broadcast message, node $\mathrm{j}$ compares its distance to each cluster head, in accordance with the principle of proximity to join any cluster, and reply to joining a cluster request to the cluster head. 


\section{Simulation Analysis}

\section{Experimental parameters}

Table 2 simulation parameters table

\begin{tabular}{|l|c|l|c|}
\hline parameter & value & parameter & value \\
\hline Regional network & $100 * 100$ & The free space model (Emp) & $0.0013 \mathrm{pJ} / \mathrm{bit} / \mathrm{m} 2$ \\
\hline Sensor node number (N) & 100 & Multipath fading model (Efs) & $10 \mathrm{pJ} / \mathrm{bit} / \mathrm{m} 4$ \\
\hline The Sink node position & $(50,150)$ & Data fusion energy (EDA) & $5 \mathrm{~nJ} / \mathrm{bit} / \mathrm{signal}$ \\
\hline The cluster head expected probability (q) & 0.1 & The control packet size (cPL) & $200 \mathrm{bit}$ \\
\hline The initial energy of node (E0) & $0.5 \mathrm{~J}$ & The packet size (PL) & $4000 \mathrm{bit}$ \\
\hline $\begin{array}{l}\text { The reception or transmission of energy } \\
\text { consumption (Eelec) }\end{array}$ & $50 \mathrm{~nJ} / \mathrm{bit}$ & & \\
\hline
\end{tabular}

\section{Analysis of simulation results}

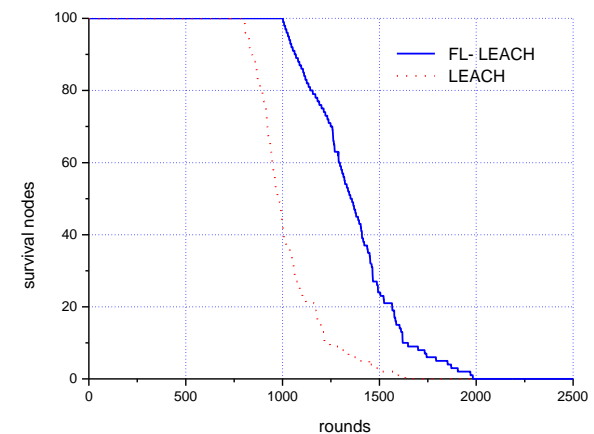

Fig. 5 alive node -round number diagram

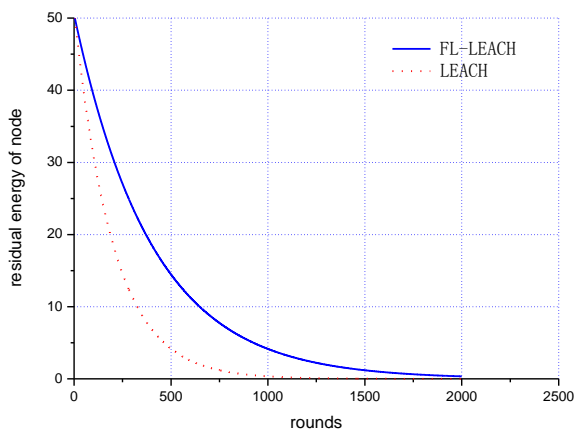

Fig. 6 residual energy - round number diagram

Fig. 5 compares the whole network life cycle of LEACH with FLCHS protocol. In the LEACH protocol, the first node dies in the 803rd round, and the last node dies in the 1162nd round,while in the FLCHS protocol, the first node dies in the 1631st round., and the last node dies in the 1984th round. From the overall trend of change curve, the death rate of alive nodes in FLCHS protocol is slower than the LEACH protocol.

Fig. 5 shows that a node with high energy, a lot of neighbor nodes and near the base station has a greater probability of elected cluster head ,So that it can balance the energy load of network, and prolong the network life cycle.

Fig. 6 shows the residual energy of LEACH network and FLCHS protocol.It is easy to find that the residual energy of network using FLCHS protocol is higher than using the LEACH protocol after the same number of rounds.Through the 200 round, 500 round, 1000 round of cycle, the residual energy of network using the FLCHS protocol is the average using LEACH protocol about 5.23 times. The residual energy of network using the FLCHS protocol tends to 0 after about the 2000th round, while after about the 1000th round using LEACH protocol.

The reason for this phenomenon is that when the node energy is low or very low, the possibility of the node become the cluster head is very low in the FLCHS protocol. So it avoids the excessive consumption of energy of nodes. However, the improved algorithm takes into account the energy of the node, location and other information.These may consume some energy. 


\section{Conclusion}

According to the deficiency of LEACH cluster head selection algorithm, this paper is fully considered in the energy factors, distribution density and the distance to the base station In the process of selecting cluster head, it uses fuzzy inference to calculate the precise probability of elected as cluster head, then uses probability value to improve threshold value by the formula.In this paper, it puts forward a kind of cluster head selection algorithm based on fuzzy logic.Through simulation and comparison with LEACH protocol,FLCHS can make the network more balanced energy consumption, prolong network life.

\section{Acknowledgements}

This work was supported by Jiangxi College of Technology, Natural Science Foundation Project (ZR12YB10)

\section{References}

[1] Wendi Rabiner Heinzelman, Anantha Chandrakasan, and Hari Balakrishnan. Energy-Efficient Communication Protocol for Wireless Microsensor Networks[J]. Proceedings of the Hawaii International Conference on System Sciences, January 4-7, 2000.

[2] Shen Xiaorui. Research on Clustering Routing Protocol Using Fuzzy Logic in Wireless Sensor Network. Taiyuan: Taiyuan University of Technology, 2010.

[3] Su Guodong, Wang Ping, $\mathrm{Xu}$ Shiwu etc.Clustering Routing Protocol for Wireless Sensor Network Based on Fuzzy Logic[J].The application of computer systems, twenty-second volume seventh issue: 117-121,2013 .

[4] Luo Yuanyuan. Research on Routing Protocol Based on Fuzzy Logic in Wireless Sensor Network[D]. Wuhan: Wuhan Institute of Technology, 2011.

[5] Gupta I, Riordan D, Sampalli S. Cluster-head Election using Fuzzy Logic for Wireless Sensor Networks. Communication Networks and Services Rearch Conference. May 2005:25 